\title{
A Greenhouse Control with Sectional-Control Strategy Based on MPT Intelligent Algorithm
}

\author{
Fengyun Wang, Lin Mei, Wenjie Feng, Lei Wang, Limin Wang, and Huaijun Ruan* \\ S\&T Information Engineering Research Center, Shandong Academy of Agricultural Sciences, \\ Jinan 250100, Shandong Province, P.R. China \\ wfylily@163. com
}

\begin{abstract}
The greenhouse are classified as complex systems such as large lag, multi-input multi-output (MIMO), non-linear and difficult to create mathematic model and so on, so it is difficult to implement classical control methods for this kind of process. A new sectional-control strategy is put forward. It uses different control modes for different deviation domains. The new strategy is based on MPT intelligent control algorithm which improves on the traditional PID algorithm and adds in self-adapting, fuzzy control, expert self-tuning etc intelligent control functions. When the deviation exceeds a certain domain, fuzzy control is used to prevent the saturated integral; when the deviation reduces to within a certain domain, MPT algorithm is used to reduce the overshooting during response process and eventually eliminate the residual. The application in greenhouse control shows that the sectional-control strategy makes the output tracing the set value correctly.
\end{abstract}

Keywords: Greenhouse, MPT, Sectional-control, Fuzzy.

\section{Introduction}

The agricultural greenhouses were used to protect the crop against the weather changes. With technical progress, the greenhouses have become a production means used to control the crop environment in order to obtain higher quality [1]. To achieve environmental conditions favorable for plant growth, greenhouses are designed with various components, structural shapes, and numerous types of glazing materials. They are operated by hand or by control system differently according to each condition.

The control object of greenhouse is the environment system which factors such as humidity, temperature, $\mathrm{CO}_{2}$ density and so on are affected not only by external climate, but also by the interaction between the environment and the plant. In control system, they are also controlled by the control components [2]. Therefore, the greenhouse system is a non-linear, multi-input multi-output (MIMO) complex system they present time-varying behaviors and they are subject to pertinent disturbances

\footnotetext{
* Corresponding author. Supported by: National science and technology support plan of P.R. China (2011BAD21B06), Science and Technology Development Plan of Shandong Province, China (2011GGC02035).
} 
depending generally on meteorological conditions [3]. All these make it difficult to describe a greenhouse with analytic models and to control them with classical controllers.

The traditional PID algorithm was widely used because it needn't know the model of controlled object. But it is difficult to realize the high control accuracy when reduce the overshooting. In our case, we put forward a new MPT algorithm which improves the PID algorithm and adds in self-adapting, fuzzy control, expert selftuning etc control functions. On the base, a new sectional control strategy is created. When the deviation is beyond a certain domain, fuzzy control rules are used to eliminate the integral saturation. When the deviation is within the domain, MPT is used to reduce the overshooting and eventually eliminate the residual. The feature of sectional strategy is that it needn't consider the accurate mathematical model of controlled object. Finally its application in greenhouse is given and the results show that it can obtain the ideal effects.

\section{MPT Algorithm}

The simplified expression of traditional PID algorithm is as follows:

Output $=$ Proportional action $(P)+\operatorname{Integral} \operatorname{action}(I)+\operatorname{Derivative} \operatorname{action}(D)$

In traditional PID control system, it is difficult to reduce the overshooting at the same time improving the control accuracy. The main reason is the integral action. If it reduces the integral action, the residual isn't easy to eliminate, and when there is disturbance, the speed to eliminate the error is slow. If it enhances the integral action, the oscillating process intensifies and it is hard to avoid overshooting. Therefore, PID algorithm is partly improved as follows:

Output $=$ Proportional action $(P)+$ Integral action $(I)+$ Derivative action

$$
(D)+\text { Derivative-integral action }\left(\int I\right)
$$

Because derivative-integral action is added in formula (2), the integral saturation is greatly improved. But we can see from formula (2) that there is a new parameter ( $\int I$ ), therefore these parameters must affect each other to make the new parameter more difficult to confirm. Through serious research and experimental analysis, the ratio between proportional action and derivative action is same to the ratio between the integral action and derivative action and the best ratio is related to the lag time of controlled object. If the lag time is longer, the response of proportional action decreases and the response of derivative action increases. The relationship is as follows:

$$
\text { Proportional action }=K(1 / t)
$$

$$
\text { Derivative action }=K(1-1 / t) d
$$


Where, $K$-Coefficient; $t$-Ratio between lag time and control period, $t \geq 1 ; d$ Derivative action. Then the formula of improved algorithm of PID is:

$$
\text { Output }=P[1 / t+(1-1 / t) d]+(1 / M) \int[1 / t+(1-1 / t) d]
$$

In formula (5), $P$ is used to regulate the derivation and proportion. When $P$ increases, it increases the derivative time and decreases the proportional band at the same time. Verse On the contrary, when $P$ decreases, it decreases the derivative time and increases the proportional band. $M$ is similar to integral time to regulate the integral action and derivative-integral action. $t$ is used to regulate the mutual proportion between derivative action and proportional action. If $t=1$, the derivative action is zero. If $M=0$, the integral action is zero.

Thus the control parameters are reduced to 3. Because the traditional PID parameters are only according to the algorithm self and its feature is that it needn't know the accurate model of controlled object. Because three improved parameters differ from the original parameters, the improved PID algorithm is defined as MPT control algorithm and the implication of three parameters as follows:

\section{$2.1 \quad M$}

M50 is retaining parameter. The definition of M50 is the difference between stable measured value when the output value is $50 \%$ and stable measured value when the output value is $0 \%$. Smaller the $\mathrm{M}$ value is and stronger the integral action is. Larger the $\mathrm{M}$ value is and weaker the integral action is (integral time adds). If $M=0$, the integral action is cancelled.

\section{$2.2 \quad \mathbf{P}$}

$\mathrm{P}$ is rate parameter. $\mathrm{P}$ is inverse proportional to the change of measured value when the output changes $100 \%$ within one control period. The definition as follows: $P=100 /$ change of controlled parameter every second. The best value of $\mathrm{P}$ is usually confirmed by self-tuning method. $\mathrm{P}$ value affects the proportional action and derivative action. Proportional action and derivative action increase proportionally with $\mathrm{P}$ value increasing. Proportional action and derivative action weaken correspondingly with $\mathrm{P}$ value decreasing. $\mathrm{P}$ is unrelated to integral action.

\section{$2.3 \quad$ T}

$\mathrm{T}$ is lag time parameter. $\mathrm{T}$ is the time required when the output rises to $63.5 \%$ of maximum value and its unit is second (s). $\mathrm{T}$ is introduced and set correctly to overcome the overshooting and oscillation completely and make the response at the best speed. T affects the proportional action and derivative action. Smaller T value is, stronger the proportional action is and weaker the derivative action is. Larger $\mathrm{T}$ value is, the proportional action weakens and derivative action enhances. If $\mathrm{T}$ is less than or 
equal to control period, the derivative action is cancelled completely and when the regulation becomes proportional or proportional-integral.

\section{$3 \quad$ Intelligent Sectional Strategy}

The intelligent control is with some intelligent characteristic as follows: (1) Integrate the human's experience with control theory. (2) With the capacity of on-line studying, modifying and creating new knowledge. (3) Deal with qualitative and quantitative information and fuzzy and accurate information. (4) With stronger logical reasoning and analytical decision-making than traditional control [4]. The intelligent control is the development of traditional control theory and combination of control theory, computer technology and artificial intelligent technology. Therefore it is with capacity of self-adapting, self-studying and self-organization[5].

\subsection{Self-adapting}

The greenhouse is a complex control object with severe non-linear characteristic and intercoupling between parameter and parameter which is solved by self-adapting control mode. The feature of self-adapting is that when the controlled deviation is larger than the estimated error, the self-adapting system doesn't modify MPT parameters but modify the output to lower the error. Though the modified range is limited, the phenomenon that original correct controlled parameters are modified to wrong value doesn't happen to make the system respond rapidly which greatly improve the control accuracy.

\subsection{Self-tuning}

In the intelligent algorithm, the traditional PID algorithm parameters have been modified to MPT parameters. In order to confirm MPT parameters, a set of selftuning expert system is introduced. Because MPT parameters are described for controlled object, its self-adapting and self-tuning are simpler than traditional PID parameters and accurate. In general case, if the operation of self-tuning is correct, its success rate is almost $100 \%$.

The process of self-tuning uses position control to regulate the system. After the oscillation, the lag time parameter $\mathrm{T}$ is confirmed by the period and the rate parameter $\mathrm{P}$ is confirmed by oscillation amplitude. It isn't easy to confirm the parameter $\mathrm{M}$ directly. For temperature, it is usually assumed that the measured value is $25^{\circ} \mathrm{C}$ when the output is zero and parameter $\mathrm{M}$ is confirmed in accordance with the output when oscillating. For linear input, $M$ value is its scale range. Therefore the best values of parameter $\mathrm{M}$ and $\mathrm{T}$ can be obtained by self-tuning, but parameter $\mathrm{M}$ is only rough. In addition, if the rate parameter or lag time is very long, self-tuning may also increase the control period to make the system conform to the actual requirements of controlled object. 


\subsection{Fuzzy Control}

The classical fuzzy controller uses the fuzzy set theory to directly convert the language rules formed by expert knowledge or operator's experience into automatic control strategy (usually the fuzzy rules table) which doesn't rely on the accurate mathematic model of controlled object but use its language knowledge model to design and modify the control algorithm [6,7].

When the controlled deviation is very large, it hasn't too large significance for the output by formula regulation which inversely produces integral saturation. Though it uses MPT algorithm, if the controlled parameters are set incorrectly, the integral saturation or over-integral phenomena will also occur. Therefore when the controlled parameters are beyond the proportional band, the fuzzy control is used to confirm the output which can obtain ideal control effect.

\subsection{Sectional Strategy}

The sectional strategy adopts different control modes in the deviation domain. Its block diagram is shown in Fig. 1. When the deviation is larger than a certain domain, the fuzzy control is selected by operation mode selector to overcome integral saturation and confirm the output by fuzzy rules. When the deviation reduces within a certain domain, selector switches to MPT algorithm to decrease the overshooting during responding and eventually eliminate the residual. Therefore the sectional strategy integrates the merits of MPT algorithm and fuzzy control to improve the sensitivity and accuracy and obtain the ideal control effect.

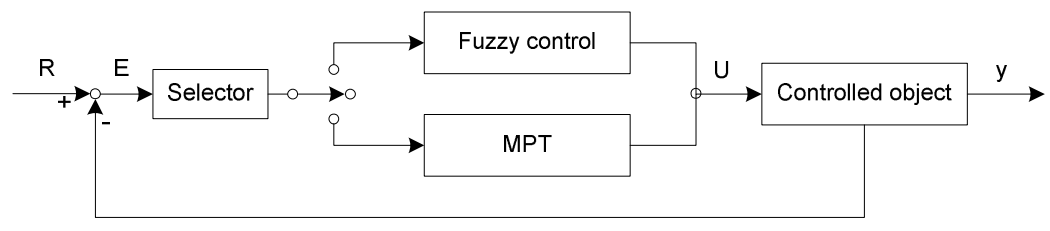

Fig. 1. Block diagram of sectional strategy

\section{Application in Greenhouse}

\subsection{Control Parameters}

The application is in a new built phalaenopsis greenhouse to effectively combine phalaenopsis planting with greenhouse control design to realize the optimal growth environment of phalaenopsis. The parameters that affect the growth of phalaenopsis are light intensity, temperature and humidity etc. The main parameters we will discuss hereinafter are light intensity and temperature. 


\subsubsection{Temperature}

There is a critical temperature, $25^{\circ} \mathrm{C}$, between the vegetative growth and reproductive growth of phalaenopsis and the critical state is during the flower spikes appearing. During the period of peduncle growing out, the temperature should be kept at $20 \sim 25^{\circ} \mathrm{C}$ in daytime and $22 \sim 18^{\circ} \mathrm{C}$ in nighttime. This period will last $20 \sim 30$ days until the peduncle grows out. The sensitivity to temperature for various phalaenopsis is various. The minimum temperature for phalaenopsis is $2 \sim 3^{\circ} \mathrm{C}$ in dry environment and $15^{\circ} \mathrm{C}$ under general humidity condition. During vegetative growth phase, the temperature is usually over $25^{\circ} \mathrm{C}$.

\subsubsection{Light Intensity}

The light needed by phalaenopsis growth is lower and continuously increases with its growth. Light intensity in various growth phases is shown in Table 1.

Table 1. Light intensity in various growth phases

\begin{tabular}{cccccc}
\hline Growth phase & $\begin{array}{c}\text { Bottle } \\
\text { seedling }\end{array}$ & Little seedling & $\begin{array}{c}\text { Middle } \\
\text { seedling }\end{array}$ & Big seedling & Mature plant \\
\hline $\begin{array}{c}\text { Light intensity } \\
\text { (lux) }\end{array}$ & $5000 \sim 7000$ & $7000 \sim 10000$ & $10000 \sim 15000$ & $15000 \sim 20000$ & 20000 40000 \\
\hline
\end{tabular}

\subsubsection{Humidity}

The requirements of humidity for seedling and flowering of phalaenopsis are various. The ideal humidity during the period of seedling is from $70 \%$ in daytime to $90 \%$ in nighttime. During this period, the higher the humidity is and the quicker the seedling grows but easier the germ grows. The ideal humidity during the period of mature plant is from $60 \%$ in daytime to $80 \%$ in nighttime.

\subsection{Control System}

\subsubsection{Hardware}

The system is composed of measuring loop, controller and output loop. It may include one computer and printer depending on the requirements. In according to the production requirements, the measuring loop mainly measures the light intensity, temperature and humidity. So the sensors are light intensity sensor, indoor and outdoor air temperature sensors, soil temperature sensor, and indoor and outdoor air humidity sensor and soil humidity sensor. The controller mainly collects the signals from the sensors, then carries out A/D converting and analysis at last makes decision to the output loop, drives the relay after D/A converting so as to control the control equipments and adjust the environment conditions. The output loop mainly includes the inner and outer shade net motors, side window motor, wet curtain motor, fan, circulating motor and spray irrigation trolley. 


\subsubsection{Software}

The system adopts MPT intelligent algorithm based sectional strategy. The develop environment for controller is Windows $2000+$ KeilC7.01 + Wave + Labtool48. The operating enviroment is W77E58 + 32KBFlash+watchdog+extending parts. The software realizes the parameters set, data and time display, alarm information hint and online state indication etc[8].

\section{$5 \quad$ Application and Conclusion}

The system was installed in the phalaenopsis greenhouse in Jining Agricultural Hitech Demonstration Zone. The ideal values of parameters are set in according to the phalaenopsis growth model [9] and expert knowledge library[10]. Fig. 2 is the comparison curve between actual measured values by the indoor and outdoor air temperature sensors and the ideal value from zero clock on $24^{\text {th }}$, Sep., 2006 to zero clock on $25^{\text {th }}$, Sep., 2006. Fig. 3 is the comparison curve between actual measured values by the indoor and outdoor light intensity sensors and the ideal value from zero clock on $24^{\text {th }}$, Sep., 2006 to zero clock on $25^{\text {th }}$, Sep., 2006.

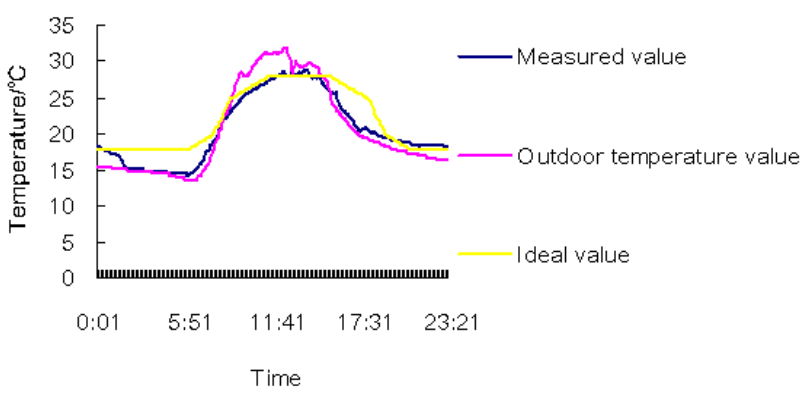

Fig. 2. Comparison curves between the measured temperature in greenhouse, outdoor temperature and ideal value

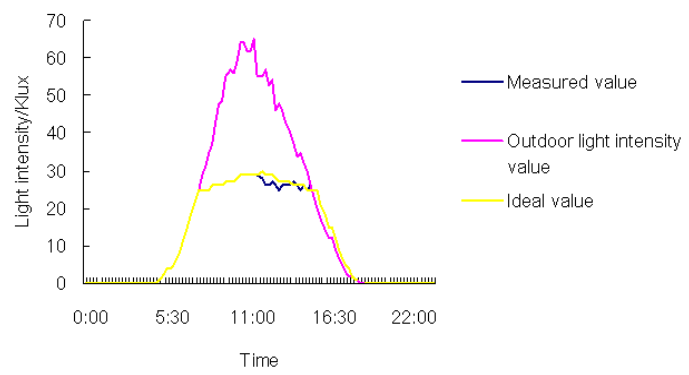

Fig. 3. Comparison curves between the measured light intensity in greenhouse, outdoor light intensity and ideal value 
When it didn't take warming measures, the greenhouse only kept the inner temperature by the heat preservation of itself in night and adjusted its temperature by side window, wet curtain and fan etc. It didn't take supplementary lighting measure in night and controlled the light intensity by inner and outer shade net. We can see from the curve that the sectional strategy based system can trace the set values well.

\section{References}

1. Trabelsi, A., Lafont, F., Kamoun, M., Enea, G.: Fuzzy identification of a greenhouse. Applied Soft Computing 7, 1092-1101 (2007)

2. Wang, D.: SVM Regression Modeling for Greenhouse Environment. Transactions of the Chinese Society for Agricultural Machinery 35(5), 106-109 (2004)

3. El Ghoumari, M.Y., Tantau, H.-J., Serrano, J.: Non-linear constrained MPC: real-time implementation of greenhouse air temperature control. Computers and Electronics in Agriculture 49, 345-356 (2005)

4. Wu, H., Xie, Y., Li, Z., He, Y.: Intelligent control based on description of plant characteristic model. ACTA Automatica Sinica 25(1), 9-17 (1999)

5. Fourati, F., Chtourou, M.: A greenhouse control with feed-forward and recurrent neural networks. Simulation Modelling Practice and Theory 15, 1016-1028 (2007)

6. Zhang, H., Yang, Y., Chai, T.: Current state and development multi-variable fuzzy control (I). Control and Decision 10(3), 193-203 (1995)

7. Trabelsi, A., Lafont, F., Kamoun, M., Enea, G.: Fuzzy identification of a greenhouse. Applied Soft Computing Journal 7(3), 1092-1101 (2007)

8. Fitz-Rodríguez, E., Kubota, C., Giacomelli, G.A., Tignor, M.E., Wilson, S.B., McMahon, M.: Dynamic modeling and simulation of greenhouse environments under several scenarios: A web-based application. Computers and Electronics in Agriculture 70, 105-116 (2010)

9. Zhang, X., Liu, F., Wang, F., Liu, S., Feng, W.: Design of implementation on dynamic vegetation model of greenhouse phalaenopsis. Chinese Agricultural Science Bulletin 23(11), 398-402 (2007)

10. 2007SR15256.2007-2-1 